\title{
ORIGEN ÉTNICO Y CAMBIOS INTERGENERACIONALES EN LOS NIVELES EDUCACIONALES Y SOCIALES EN LA ARAUCANÍA
}

\author{
GUILLERMO WILLIAMSON \\ Profesor Asociado e Secretario Académico del Departamento de Educación \\ de la Universidad de La Frontera - Temuco, Chile \\ gwilliam@ufro.cl
}

\section{VIOLETA CANTERO}

Académica de la Facultad de Ingeniería, Ciencias y Administración e Jefe del Área de Economía del Departamento de Administración y Economía, de la Universidad de La Frontera - Temuco, Chilevcantero@ufro.cl

\section{RESUMEN}

El artículo hace referencia a los cambios intergeneracionales que se han dado, tanto en términos educacionales como por categorías sociales, en tres de las principales comunas de la Región de la Araucanía, Chile. En él se analizan las tasas de movilidad educacional y la incidencia de la educación de los padres (capital educacional) en el nivel educacional de los hijos. Además, se muestra la transformación social intergeneracional por origen étnico que se ha dado en la región, así como las mayores oportunidades ocupacionales de los individuos que han accedido a los niveles superiores de enseñanza. El estudio pone de manifiesto que aun cuando siguen existiendo inequidades educativas por origen étnico, la educación superior ha abierto oportunidades de ascenso social para la mayoría de los individuos que han accedido a ella.

CLASE SOCIAL - CHILE - MOVILIDAD DEL ALUMNO - RAÇA

\section{ABSTRACT}

INTERNATIONAL CHANGES IN THE EDUCATIONAL AND SOCIAL LEVELS OF PEOPLE OF ETHNIC ORIGIN IN THE AURACANIA REGION. This article refers to the inter-generational changes that have occurred in educational terms and by social category in three of the main communes of the Araucária region of Chile. It analyses the rates of educational mobility and the incidence of parent's education (educational capital) in the educational level of the children. It also provides evidence of the social and inter-generational transformation by ethnic origin that has occurred in this region, as well as of the greater occupational opportunities opened for individuals who have risen to higher levels of education. The study shows that even when educational inequality resulting from ethnic origin remained, higher education has created opportunities for social mobility for most those who had access to it.

SOCIAL CLASS - CHILE - STUDENT MOBILITY - RACE 
Las cifras generales existentes en el tema educacional evidencian que el país ha logrado importantes avances; sin embargo y pese a ello persisten desigualdades educacionales y sociales entre los individuos de mayores ingresos y los de menores recursos, planteándose no sólo deudas de calidad sino también y cada vez más, de equidad (OCDE, Chile, 2004). Esta desigualdad social también se manifiesta al comparar la población indígena con la no indígena. La población indígena en Chile, como en otros países del mundo, experimenta con especial rigor situaciones de pobreza y exclusión social (Chile, 2002). En el país y en la IX Región de La Araucanía existen desigualdades en ingresos y educación que afectan significativamente a la población indígena, observándose significativas diferencias en materia de ingresos y educación entre indígenas y no indígenas (Chile, 2002).

Según Winkler y Cueto (2004), la desigualdad en las oportunidades educativas comienza en la familia y el hogar, especialmente por su ubicación urbano/ rural y el nivel de educación de la madre, que afectan la nutrición y la salud del niño, al igual que su disposición a ingresar a la escuela y la probabilidad de desertar, además de afectar la cantidad de conocimientos que el niño adquiere mientras está en la escuela. En este sentido y por su condición de desigualdad inicial, los niños indígenas comienzan su educación escolar con una grave desventaja en relación con los niños no indígenas (Unicef, Chile, 2003).

En el caso de la Región de la Araucanía, el tema de la desigualdad educacional y de ingresos es una cuestión preocupante por dos razones: primero, esta región es una de las zonas geográficas del país que presenta los más altos índices de pobreza e indigencia, y segundo, es una de las regiones con mayor concentración porcentual de grupos étnicos, en especial de población mapuche. Por otra parte, la región concentra sus principales actividades económicas en la agricultura, la ganadería y el área forestal, actividades que en gran parte se encuentran en manos de campesinos y comunidades indígenas que viven en la ruralidad. Para estas familias acceder a una educación urbana y de buen nivel no es fácil, lo que obliga que sus hijos muchas veces abandonen los estudios tempranamente, con los consiguientes efectos negativos que ello conlleva, manteniéndose así las desigualdades educacionales y sociales.

Al igual como ha sucedido a nivel país, la región muestra mejoras sistemáticas en el tema educacional; sin embargo, poco se sabe cómo estos mayores logros educacionales han sido aprovechados por los diferentes segmentos 
socioeconómicos y, en especial, por los grupos étnicos. Este estudio aporta antecedentes de cómo se ha dado el proceso de movilidad educacional intergeneracional por origen étnico en tres de las principales comunas de la Región de la Araucanía, y de qué forma ello ha impactado en términos de oportunidades ocupacionales y de bienestar social. Lo anterior es importante, dado que hoy en día, para ascender socialmente, ya no basta con acceder a la educación básica y media, sino que se requiere una formación de nivel profesional superior, ya sea técnica o universitaria, que permita que los individuos opten por mejores puestos de trabajo, y por ende, por mayores ingresos.

Este artículo se refiere a los hallazgos de un proyecto de investigación sobre movilidad social y educación de la Facultad de Ingeniería, Ciencias y Administración, cuyos resultados son complementados y comentados con aprendizajes de otro proyecto de investigación sobre Educación Intercultural Bilingüe - EIB - de la Facultad de Educación y Humanidades, ambos de la Universidad de la Frontera'; la principal información metodológica y los resultados corresponden al primer proyecto. En el texto usaremos indistintamente los conceptos de etnia y pueblo para referirnos al pueblo mapuche.

\section{ASPECTOS METODOLÓGICOS}

La información que aquí se proporciona proviene de una encuesta realizada a una muestra de 500 jefes de hogar en 3 de las principales comunas de la Región de la Araucanía: Temuco, Padre las Casas y Nueva Imperial, para lo cual se empleó el método de muestreo irrestricto aleatorio para proporciones $^{2}$. Los jefes de hogar entrevistados fueron hombres y mujeres entre

I. Proyectos de Investigación nanciados por la Universidad de la Frontera: Educación y Movilidad Social: Un Estudio Empírico Aplicado a las Familias Mapuche y No Mapuche de las Comunas de: Temuco, Padre las Casas y Nueva Imperial, que coordina la académica Violeta Cantero y Estado del Arte y Evaluación de la Situación Actual de la Educación Intercultural Bilingue, que coordina el académico Guillermo Willianson.

2. La muestra corresponde a la fórmula del " $n$ " o el tamaño de muestra requerido para las proporciones p y q; con un límite B para el error de estimación es:

$$
\eta=\frac{N p * q}{(N-1) D+p * q}=\frac{92.988 *(0.5) *(0.5)}{92.987 *(0.000625)+(0.5) *(0.5)} \cong 400 \text { encuestas. }
$$


25 y 65 años de edad, de procedencia mapuche y no mapuche, habitantes de la zona urbana y rural de estas comunas ${ }^{3}$. La edad de 25 años para los entrevistados tiene relación con el límite de edad en que finaliza la educación formal - incluyendo la superior - para la mayoría de la población.

El instrumento de recolección de datos fue diseñado para vincular características de los miembros de una generación (los padres) con los niveles educacionales que alcanzan los miembros de la generación siguiente (sus hijos). De esta forma se puede poner de manifiesto el peso que tienen tanto el ingreso familiar como la educación de los padres (el capital educacional del hogar) en la determinación de los años de educación que alcanzan los jóvenes.

Esta encuesta fue aplicada en el período comprendido entre agosto de 2005 y enero de 2006. Las 500 encuestas realizadas se distribuyeron proporcionalmente en las tres comunas en estudio, considerando el número de jefes de hogar en las zonas urbanas y rurales (36I encuestas en Temuco, 82 en Padre las Casas y 57 en Nueva Imperial). En las zonas urbanas, las encuestas se aplicaron buscando representatividad geográ ca de todas las localidades, villas o sectores;

Donde $\mathrm{N}$ corresponde al total de jefes de hogar (hombres y mujeres) de las comunas en estudio, que ascendió a 92.988 personas. Para ampliar el tamaño de la muestra se trabajó con máximas proporciones: $p=0.5 ; q=0.5$ ( $50 \%$ hombres y $50 \%$ mujeres), a pesar de que los datos arrojaban un $70 \%$ hombres y $30 \%$ mujeres. En este caso, se consideró un error de estimación de 5\% con un 95\% de con anza, de lo cual se obtuvo que: $\mathrm{D}=\mathrm{B}^{2} / 4_{-}=(0.05)^{2} / 4=0.000625$.

La fórmula empleada arrojó un " $n$ " aproximado de 400 encuestas, pero para reducir el error de estimación y, dados los objetivos del estudio, la muestra se amplió a 500 encuestas.

3. Debido a la creciente participación de la mujer en la fuerza de trabajo y en las jefaturas de hogar, este estudio incluye tanto a jefes de hogar hombres como mujeres. Al respecto, datos de la encuesta Caracterización Socieconómica Nacional - Casen - 2003 reflejan que la tasa de participación de las mujeres en la fuerza de trabajo experimentó un signicativo crecimiento de 2,7 puntos porcentuales, pasando de 39,3\% a 42,0\% entre los años 2000 y 2003. Este aumento se explica por la incorporación, en el período, de cerca de 300 mil mujeres al mercado del trabajo. Además, los datos poblacionales del Censo 2002 muestran una reducción del porcentaje de casados y un incremento en la tasa de conviviente-pareja y en la tasa de separados-anulados, lo que ha hecho necesaria una mayor independencia femenina en términos laborales y una mayor participación femenina como jefas de hogar, motivos más que su cientes para no discriminar respecto a sexo. 
en tanto en las zonas rurales primó la facilidad de acceso y las cercanías de las viviendas entre los sectores entrevistados.

\section{LA EDUCACIÓN COMO FACTOR IGUALADOR DE LAS OPORTUNIDADES SOCIALES}

En el tema de la movilidad ocupacional y social, la educación juega un importante rol, puesto que permite que los individuos se trasladen de un nivel socioeconómico a otro a través del conocimiento y especialización que ésta proporciona. Según Hopenhayn (2003), la educación se puede considerar como un eslabón privilegiado para articular la integración cultural, la movilidad social y el desarrollo productivo. Una sociedad con altos niveles de escolaridad y buenos logros educativos debería tender a ser más igualitaria en su estructura de ingresos (mediante la rentabilidad laboral de la educación) y por ende podría crecer mediante aumentos en la productividad y no en virtud de la sobreexplotación de recursos humanos o naturales.

En la actualidad existe acuerdo en que el sistema educacional juega un papel central en la formación de capital humano, entendiendo por éste el conjunto de habilidades y conocimientos de que dispone una persona para desempeñarse en las actividades productivas (Pérez Brignoli, 2004), y lo que en palabras de Corbo (2002) implica preparar estudiantes con la capacidad y creatividad necesarias para que, una vez que se integren a la fuerza laboral, sean capaces de operar eficientemente el stock de capital que incorpora las nuevas tecnologías. Por ende, el principal desafío es mejorar la cobertura y la calidad de la enseñanza en todos los niveles de la educación formal, para así construir los cimientos de un sistema educativo que contribuya al crecimiento y a la mejora de la distribución del ingreso. En este mismo sentido, Pérez Brignoli (2004) señala que si bien los contenidos de la educación son fundamentales en la formación de capital humano, el tema es aún más complejo, porque existen otros factores que lo afectan y que tienen que ver con el contexto cultural e institucional en el que se desenvuelven las personas. Según este autor, la educación formal acumulada resulta ser un componente fundamental (y relativamente fácil de observar y medir) pero de ningún modo exclusivo en la formación de capital humano. La noción va estrechamente vinculada a la productividad y rendimiento del trabajo humano e incluye por lo tanto varios componentes 
distintos: a) la educación formal; b) el aprendizaje práctico (learning by doing) a lo largo de la vida; c) los entrenamientos posteriores a la graduación formal; y d) el estado de salud física y emocional.

Según Germani (1961), la educación puede estimular la movilidad social no solamente entregando conocimientos básicos y específicos, sino que también fomenta la movilidad mental a través de la internalización de ciertas actitudes; por ejemplo, los sistemas educativos pueden enseñar las personas a trabajar en equipo, a ser innovadoras y líderes, formando un tipo de individuos que son móviles psicológicamente y en general tienen mayores posibilidades de movilizarse socialmente; también la educación puede promover la movilidad ocupacional, aumentando los niveles de aspiración y las actitudes competitivas requeridas en el ámbito laboral, lo cual podría tener como consecuencia una movilidad social.

Desde esta perspectiva, la educación constituye uno de los pilares fundamentales para lograr que una sociedad democrática se desarrolle en igualdad de condiciones y que las personas adquieran estándares de vida adecuados. En general, se puede decir que el nivel de educación de un individuo condicionará en gran medida el tipo de trabajo al cual pueda acceder y el salario asociado a dicha actividad. Tomando como referencia la relación existente entre educación e ingreso de las personas, se debería esperar que a mayor grado de educación los individuos adquieran una más amplia capacidad de respuesta frente al mercado laboral, factor que parece ser determinante para lograr una mayor productividad, la cual a su vez permitirá alcanzar adecuados niveles de ingresos en el mercado laboral. Estudios de Mideplan (Chile, 2003) evidencian que los cambios más significativos en los ingresos se comienzan a percibir a partir de los 12 años de estudio (enseñanza media completa; actualmente, la cantidad de años obligatorios que constituyen el derecho a la educación). Una vez que el individuo se encuentra cursando la educación superior, con más de 12 años de escolaridad, cada año adicional de educación superior genera un alto impacto en el ingreso percibido por el individuo, alcanzando los más altos ingresos las personas que han finalizado su educación superior ( 17 y más años de escolaridad $)^{4}$. Por lo tanto, está claro

4. En Chile, si un profesional con título universitario recibe mensualmente, en promedio, un sueldo de \$1.055.000 (U\$1.955; dólar de 31.07.2006), el de un técnico de instituto 
que una persona con mayor nivel educacional puede acceder a un mayor nivel de ingreso y por ende a un mayor bienestar social en relación a una persona con un menor nivel educacional.

Sin embargo, reconociendo la importancia de la educación en la movilidad ocupacional y social, no se puede desconocer el hecho de que si bien la educación no asegura la movilidad ocupacional y social por sí misma, ni elimina la desigualdad social ${ }^{5}$, no hay oportunidad sin educación, pues tal como lo postula Filgueira (200I), las redes sociales y familiares también son importantes, además de otras características propias de los individuos. Unesco (1992) ha planteado la determinación de las economías tradicionales en la menor educación y menores ingresos, señalando que cambios en los ingresos requieren cambios educacionales, pero también en las estructuras productivas locales. Por lo tanto, a pesar de que la educación no es suficiente para fomentar la movilidad ocupacional y social, es una condición necesaria.

De lo anterior se puede concluir que - en contextos de modernización sistemática - la educación y por lo tanto la acumulación de capital humano, el que se valoriza y revaloriza en el tiempo, con los años de estudio aprobados y la experiencia acumulada, junto con el capital innato de los individuos y las redes sociales, son los principales factores que pueden generar la movilidad ocupacional y social a mediano y largo plazo, lo que además de ayudar las personas a superar la pobreza, tiene un efecto distributivo significativo.

profesional es de $\$ 513.000$ ( $\cup \$ 951$ ), de un egresado de enseñanza media es de $\$$ 257.400 ( $\ \$ 477$ ) y de básica de \$ 174.077 (U\$ 322) (Alamos, 2006, p.26).

5. Un ejemplo es la situación de la educación superior. Hoy casi I de cada 3 personas entre 18 y 24 años están en educación superior; para el año 2010 se proyecta una matrícula de 817.420 alumnos en este nivel, de los cuales 572.35 I estarían en el sistema universitario (el resto estaría en instituciones formadoras de técnicos superiores: Centros de Formación Técnica-CFTs: 91.077, e Institutos Profesionales-IPs: 153.993. Pero, si entre 1990 y 2003, la cobertura de educación superior aumentó de 16 \% a 37,5\%, según la encuesta Casen-2003, en el mismo período la cobertura pasó entre los hogares más pobres de 4,5\% a 14,5\% y entre los jóvenes de los hogares más ricos de 40,2\% a 73,7\%; es decir, en los últimos años la brecha se amplió de 35,8\% a 59,2\%. Un joven del quintil más pobre tiene hoy cinco veces menos posibilidades de entrar a la educación superior que otro del quintil más rico (Williamson, 2006). 


\section{CAMBIOS INTERGENERACIONALES EN LA ESTRUCTURA SOCIAL}

En todas las sociedades humanas se distinguen diferencias entre las capacidades o características individuales de sus componentes y aquellas que aluden a su posición social. Estas diferencias se hacen más marcadas en las sociedades tecnológicamente más complejas, donde se acentúa la división del trabajo y la diferenciación de funciones (Kerbo, 2003). La diferenciación social establece el escenario para la desigualdad y la estratificación social.

El análisis de la estratificación social y sus cambios en el tiempo (movilidad social) se puede realizar desde diversas perspectivas, pero uno de los elementos más utilizados como herramienta de estudio es la ocupación. La agrupación de los individuos en categorías sociales según su ocupación permite una determinada visión de la estructura social. Según Torche y Wormald (2004), la elección de la ocupación se funda en que el trabajo es el principal recurso que tienen y movilizan los hogares para acceder al ingreso y al bienestar material, es decir, es lo que define las oportunidades de vida presentes y futuras para la gran mayoría de la población; y además porque la ocupación es un "proxy" relativamente adecuado del acceso de las personas a la educación y al ingreso, que son dos dimensiones que subyacen y definen en un sistema de estratificación social, y que, por ende, determinan un cierto estatus o prestigio ocupacional.

Por otro lado, en la sociedad industrial moderna, la estructura ocupacional puede servir como base para realizar una "estructura de clase", con la cual se pueden construir esquemas de clases ocupacionales (Atria, 2004). Entre este tipo de esquemas se puede mencionar el de Torche y Wormald (2004) (Cuadro I). El esquema está basado en la estructura de clases planteada por Erikson y Goldthorpe ( 1993), investigadores que han realizado estudios sobre estructura social, estratificación y movilidad en Gran Bretaña, basándose en un esquema clasificatorio que deriva de una base teórica sociológica.

6. La estructura de clases se deriva de la subdivisión de la población en un cierto número de grupos distintos, en términos de recompensas materiales (Atria, 2004) y en consecuencia, para este estudio, no se considera la clase bajo un prisma marxista. 
ESQUEMA DE CLASES DE TORCHE Y WORMALD

\begin{tabular}{|c|c|}
\hline Clase o Categoría social & Categorías que incluye \\
\hline I. Clase de servicio & $\begin{array}{l}\text { Directivos, administradores, profesionales y } \\
\text { propietarios de grandes y medianas empre- } \\
\text { sas. Profesionales bajos, técnicos superiores, } \\
\text { supervisores de trabajadores no manuales y } \\
\text { administradores de empresas pequeñas }\end{array}$ \\
\hline II. Clase de rutina no manual & $\begin{array}{l}\text { Trabajadores no manuales en administraci- } \\
\text { ón, ventas y servicios }\end{array}$ \\
\hline III. Clase de pequeña burguesía & $\begin{array}{l}\text { Propietarios de empresas chicas (menos de } \\
\text { I0 trabajadores) no agrícolas }\end{array}$ \\
\hline IV. Clase de trabajadores independientes & Trabajadores por cuenta propia \\
\hline V. Clase de trabajadores manuales cali cados & $\begin{array}{l}\text { Técnicos bajos, supervisores de trabajadores } \\
\text { manuales y trabajadores manuales cali ca- } \\
\text { dos }\end{array}$ \\
\hline VI. Clase de trabajadores manuales no cali cados & Trabajadores manuales semi y no cali cados \\
\hline VII. Pequeños propietarios agrícolas & Pequeños propietarios agrícolas \\
\hline VIII. Trabajadores agrícolas & Trabajadores y peones agrícolas \\
\hline
\end{tabular}

Fuente: Torche y Wormald (2004).

El esquema de Torche y Wormald (2004), posee 8 clases sociales, las que según estos investigadores permiten una apropiada descripción de la estructura de la estrati cación social chilena. Por ejemplo, este tipo de clasi cación permite distinguir entre trabajadores no cali cados urbanos y rurales, o bien entre un contador que tiene su propia microempresa versus otro que trabaja como empleado. A pesar de que estas personas tengan la misma educación o ingresos, se insertan en la estructura social de forma diferente y enfrentan oportunidades también distintas.

Aplicando el esquema de clases ocupacionales de Torche y Wormald a la muestra del estudio, se puede ver la transformación intergeneracional que ha experimentado la estructura social en las comunas en estudio (Tab. 2): 
TABELA 2

CAMBIOS EN LA ESTRUCTURA SOCIAL: COMPARACIÓN ENTRE PADRES E HIJOSTOTAL COMUNAS, IX REGIÓN

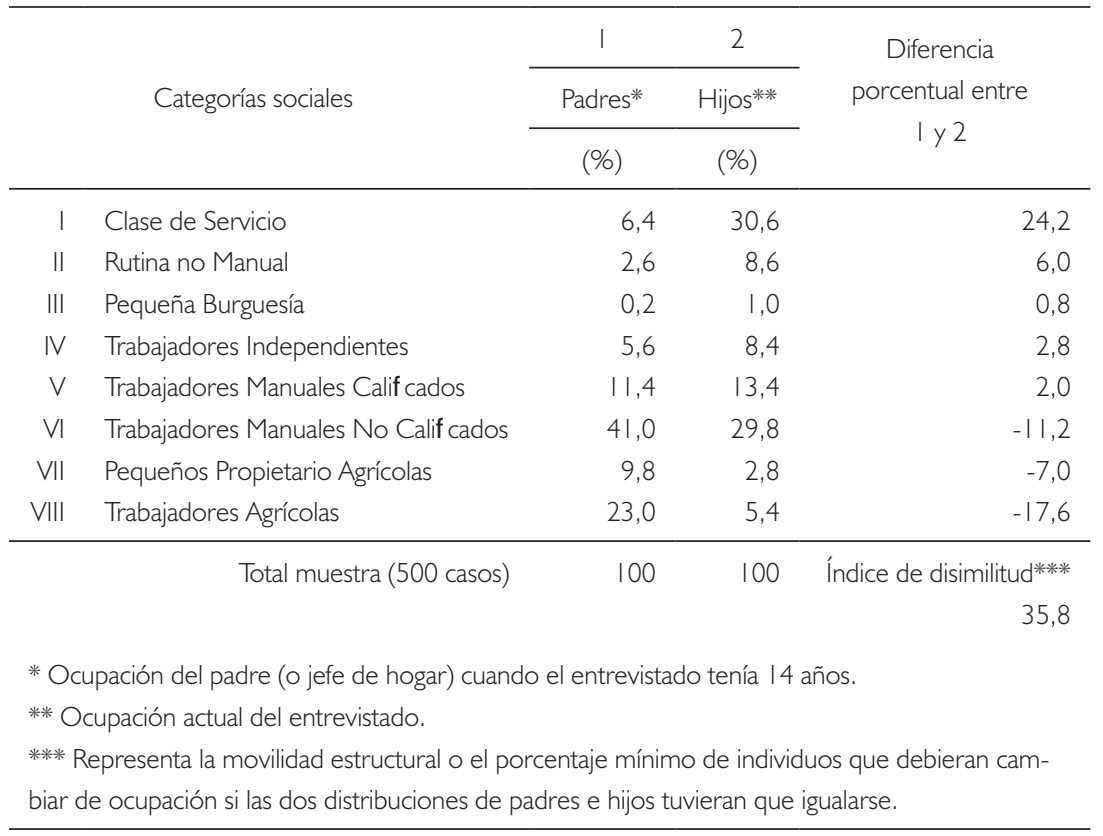

Fuente: Elaboración propia (Cantero, 2005/2006).

Como se puede ver en la tabela 2, han existido cambios significativos desde el origen (padre) hasta el destino (hijos). Las primeras 5 clases (partiendo desde la Clase de Servicio hasta Trabajadores Manuales Calificados) han aumentado su importancia relativa entre generaciones. En tanto, han perdido participación las clases de Trabajadores Manuales No Calificados, Pequeños Propietarios Agrícolas y Trabajadores Agrícolas. Estos cambios se recogen en el Índice de Disimilitud, que asciende a 35,8\%.

Al igual como lo postulan otros estudios, los datos reflejan una migración masiva desde las clases agrícolas y de escasa calificación hacia clases ocupacionales urbanas de mayor calificación. Según Torche y Wormald (2004), este hecho estaría evidenciando una pérdida de importancia relativa de la "campesinización". Esta situación también se visualiza en los datos del último Censo 
realizado en Chile (Chile, 2002), donde la población urbana se incrementó en 3,1\% entre 1992 y 2002, reduciéndose en igual proporción la densidad poblacional en la zona rural?.

Las clases que han aumentado su importancia relativa son en general clases que requieren un cierto nivel de educación y preparación para pertenecer a ellas. Por lo tanto, estos cambios de estructuras ocupacionales están en directa relación con los cambios educacionales y la movilidad educacional observada entre generaciones.

\section{MOVILIDAD EDUCACIONAL INTERGENERACIONAL}

Como es sabido, la situación del país en cuanto a educación ha experimentado signos de crecimiento en comparación con resultados de años anteriores. Las cifras muestran que el promedio de escolaridad de la población de 15 años y más aumentó de 9,0 a 10,2 años, desde 1990 hasta 2003. Entre el 2000 y el 2003, la escolaridad experimentó un importante crecimiento, pasando de 9,8 a 10,2 años de escolaridad promedio (Chile, 2003). Las cifras evidencian que los cambios en la escolaridad de la población han favorecido especialmente a los jóvenes de los sectores de menores recursos.

En el caso de la IX Región de la Araucanía, los índices de escolaridad son menores en comparación con los del país. A nivel país se tiene 10,2 años promedio de escolaridad de la población de 15 años y más, en tanto en la IX Región sólo alcanza a 9, I años (2003). Sin embargo, los años promedio de escolaridad para la Novena Región han ido aumentando con el tiempo, ya que para 1990 se tenía un promedio de 7,8 años, en el año 2000 de 8,8 años y de 9,1 el 2003. Al efectuar un análisis por zonas urbanas y rurales, la IX Región también muestra índices menores que a nivel país, alcanzando un promedio de 10,2 años para el área urbana y 6,5 años para la zona rural en comparación con 10,6 y 7,I años, respectivamente, para el país (Chile, 2003).

7. Hasta 1930 predominaba la población rural, que en I 875 llegaba al 65, I \% y en | 920 al 53,6\%. A contar de 1940, la situación se invierte: el Censo de 1992 muestra que la población rural sólo representa el 16,5\% de la población total del país y en el año 2002 esta población se reduce a un I4\% nacional, aunque en Regiones como La Araucanía, alcanza a un 34 \%. 
Sin embargo, la IX Región, si bien ha experimentado signos de mejoramiento del nivel educacional en estos últimos años, aún continúa presentando los índices de pobreza más altos del país. Según datos de la encuesta Casen 2003, existe un 29,7\% de hogares en situación de pobreza. De ellos, un 9,5 son indigentes y un $20,2 \%$ son pobres no indigentes. En la zona rural la incidencia de la indigencia es más alta que en la zona urbana, mientras que la incidencia de la pobreza no indigente es mayor en la zona urbana que en la zona rural.

Es necesario estudiar la movilidad educacional para vislumbrar lo que se debería esperar en los que se refiere a cambios ocupacionales y de movilidad social. En términos simples, la movilidad educacional corresponde al cambio en el nivel de estudios que presentan los individuos en relación al de sus padres. Este cambio puede ser: a) ascendente: el individuo logró un mayor nivel educacional que sus padres; b) descendente: el individuo alcanzó un nivel educacional inferior al de sus progenitores; o c) inmóvil: el individuo alcanza el mismo nivel educacional que sus padres.

A medida que la sociedad chilena evoluciona, los padres están cada vez más conscientes de la importancia de la educación para escalar socialmente y, por lo mismo, buscan que sus hijos logren niveles educacionales superiores a los suyos. Los estudios relativos a este tema muestran que el nivel educacional de los padres es determinante en el nivel educacional que alcanzan los hijos, puesto que mientras mayor sea el nivel educacional de los padres más posibilidades tienen los hijos de alcanzar niveles superiores de enseñanza. En esta temática, Pérez Brignoli (2004) postula que cuando los jefes de hogar no tienen educación, las oportunidades de sus hijos son notablemente menores que cuando los primeros han alcanzado algún nivel de preparación. En consecuencia, en términos de política educativa y social, educar a las personas sin educación tendría un efecto multiplicador de notable importancia.

En el caso de este estudio, los datos relativos a educación se presentan en la tabela 3. En él se puede ver la distribución relativa de las frecuencias "padres-hijos", lo que permite detectar las oportunidades de movilidad educacional de individuos con distinto nivel educacional de origen (educación del padre). La diagonal de color de la tabela pone de manifiesto los individuos que permanecen inmóviles educacionalmente o que mantienen el nivel educacional del padre. Por lo tanto, si no hubiera en lo absoluto movilidad educacional, es decir, si el nivel educacional del padre determinara totalmen- 
te el nivel educacional del hijo, se debería esperar que todos los casos se distribuyeran en la diagonal de la tabela. Claramente, ésta no es la situación, y de hecho tan sólo 103 personas (21,4\% de los encuestados) mantienen el nivel educacional del padre. Por el contrario, las cifras muestran que la gran mayoría de los entrevistados (353 personas o el 73,4\% de los encuestados) se ubica en la zona superior derecha de la tabela, lo que evidencia movilidad social ascendente o un nivel educacional superior al de su padre. Por su parte, en la zona inferior izquierda se encuentran las personas que descienden educacionalmente lo que en este caso correspondió tan sólo al 5,2\% de los encuestados (25 personas). Esto evidencia que la movilidad educacional en las comunas en estudio es bastante acentuada, especialmente la ascendente.

\section{TABELA 3}

MOVILIDAD EDUCACIONAL. DISTRIBUCIÓN DE LAS FRECUENCIAS PADRES E HIJOS COMUNAS EN ESTUDIO, IX REGIÓN

\begin{tabular}{lllllllllll}
\hline Padre Hijo & $\begin{array}{l}\text { Sin } \\
\text { Educac. }\end{array}$ & $\begin{array}{l}\text { Básica } \\
\text { incomp. }\end{array}$ & $\begin{array}{l}\text { Básica } \\
\text { completa }\end{array}$ & $\begin{array}{l}\text { Media } \\
\text { incomp. }\end{array}$ & $\begin{array}{l}\text { Media } \\
\text { completa }\end{array}$ & $\begin{array}{l}\text { Superior } \\
\text { incomp. }\end{array}$ & $\begin{array}{l}\text { Superior } \\
\text { completa }\end{array}$ & $\begin{array}{l}\text { Total } \\
\%\end{array}$ & $\begin{array}{l}\text { Total de } \\
\text { casos* }\end{array}$ \\
\hline $\begin{array}{l}\text { Sin Educa- } \\
\text { ción }\end{array}$ & 2,9 & 37,1 & 22,9 & 11,4 & 20,0 & 0,0 & 5,7 & 100 & 35 \\
\hline $\begin{array}{l}\text { Básica } \\
\text { Incompleta }\end{array}$ & 0,0 & 15,4 & 13,2 & 23,1 & 25,3 & 6,6 & 16,5 & 100 & 91 \\
\hline $\begin{array}{l}\text { Básica } \\
\text { Completa }\end{array}$ & 0,0 & 4,7 & 7,0 & 13,2 & 33,3 & 7,8 & 34,1 & 100 & 129 \\
\hline $\begin{array}{l}\text { Media } \\
\text { Incompleta }\end{array}$ & 0,0 & 0,0 & 5,7 & 5,7 & 45,7 & 11,4 & 31,4 & 100 & 35 \\
\hline $\begin{array}{l}\text { Media } \\
\text { Completa }\end{array}$ & 0,0 & 0,8 & 0,8 & 3,9 & 24,0 & 14,0 & 56,6 & 100 & 129 \\
\hline $\begin{array}{l}\text { Superior } \\
\text { Incompleta }\end{array}$ & 0,0 & 0,0 & 0,0 & 0,0 & 12,5 & 12,5 & 75,0 & 100 & 8 \\
\hline $\begin{array}{l}\text { Superior } \\
\text { Completa }\end{array}$ & 0,0 & 0,0 & 0,0 & 0,0 & 3,7 & 13,0 & 83,3 & 100 & 54 \\
\hline $\begin{array}{l}\text { Total de } \\
\text { casos }\end{array}$ & 1 & 34 & 32 & 49 & 123 & 46 & 196 & - & $48 \mid$ \\
\hline
\end{tabular}

* Se consideraron 48 I casos, que declaran educación del jefe de hogar (96,2\% de las encuestas). En un $78,8 \%$ de los casos el jefe de hogar era el padre, en un 18\% era la madre y en un 3,2\% era otro familiar. Fuente: Elaboración propia (Cantero, 2005/2006). 
Las cifras reflejan que la situación educacional de los encuestados (hijos) en las comunas en estudio, ha mejorado respecto a la de sus padres, lo cual obedece sin duda a la importancia de la educación que le otorgan los habitantes de estas comunas. Mientras la educación de los padres se concentra en los niveles educacionales básicos y medios (ver última columna de la Tabela 3), los niveles educacionales de los hijos se acentúan en los niveles medio y superior (ver última fila de la Tabela 3). Además, prácticamente el 100\% de los hijos ha recibido algún tipo de instrucción (tan sólo I encuestado declaró no tener estudios), en tanto que un 7,3\% de los padres es analfabeto (35 personas).

Las cifras de la Tabela 3 también evidencian que cuando el padre no tiene educación, el 2,9\% de los hijos tampoco accede a educación, el 37, I \% alcanza la primaria incompleta, 22,9\% la primaria completa, un 20\% termina su enseñanza secundaria y sólo un 5,7\% finaliza su educación superior. Cuando el padre tiene educación primaria completa, el 7\% de sus hijos alcanza el mismo nivel, el 33,3\% termina su enseñanza media completa y un 34, I \% culmina su educación superior. Cuando el padre posee educación media completa, la situación es aún más alentadora, dado que un $24 \%$ de los hijos alcanza el mismo nivel, un 14\% alcanza superior incompleta y un 56,6\% finaliza su educación superior. Finalmente, cuando el padre tiene educación superior completa, el $83,3 \%$ de los hijos alcanza el mismo nivel educacional, un 13\% alcanza un nivel superior incompleto y un 3,7\% la educación media completa. En síntesis, las cifras muestran una fuerte relación entre el nivel educacional del padre y el nivel educacional del hijo.

Por lo tanto, si se quiere mejorar la distribución del ingreso, es necesario generar movilidad ocupacional y social entre generaciones (padres-hijos), lo cual requiere que los hijos accedan a niveles superiores de enseñanza, especialmente profesional universitaria, pues tal como lo plantea Carabaña (2003), los estudios superiores, y en especial los estudios universitarios, son un vehículo eficaz y no clasista de movilidad social ascendente. En su planteamiento, reconoce a la Universidad como un autobús que lleva a los mismos destinos profesionales a todos los que suben en él. En palabras de este autor, los hijos e hijas de profesionales y administrativos suben al vehículo mucho más que el resto, y los hijos de obreros agrarios mucho menos. Por lo tanto, la meta de las autoridades en el tema educacional es trabajar fuertemente para permitir 
el acceso a la enseñanza superior (y en especial a la Universidad) a todos los individuos, independientemente de su origen social.

En el caso de la Región de la Araucanía, las cifras ponen de manifiesto que el acceso a la educación superior, y en especial a la educación universitaria, está abierta para todos los orígenes sociales. Esto se puede ver en la tabela 4, que muestra la distribución de los encuestados (categoría ocupacional de destino del hijo) según la clase de origen o categoría ocupacional del padre, en donde se puede observar que más del $50 \%$ de los entrevistados que culminaron sus estudios universitarios provienen de padres que desempeñaban labores relacionadas con trabajos manuales no calificados y actividades agrícolas.

\section{TABELA 4}

NÜMERO DE PERSONAS CON ESTUDIOS UNIVERSITARIOS*, COMUNAS SELECCIONADAS, IXREGIÓN

\begin{tabular}{|c|c|c|c|c|c|c|c|c|c|c|}
\hline \multirow[t]{3}{*}{ Categoría ocupacional } & \multicolumn{2}{|c|}{ Origen*** (Padre) } & \multicolumn{8}{|c|}{ Destino*** (Hijo) } \\
\hline & \multirow[t]{2}{*}{ N. } & \multirow[t]{2}{*}{$\%$} & \multicolumn{2}{|c|}{ No mapuche } & \multicolumn{2}{|c|}{ Mapuche } & \multicolumn{2}{|c|}{ Mestizo } & \multicolumn{2}{|c|}{ Total } \\
\hline & & & $N^{\circ}$ & $\%$ & $N^{\circ}$ & $\%$ & $N^{\circ}$ & $\%$ & $N^{\circ}$ & $\%$ \\
\hline - Clase de servicio & 18 & 11,3 & 117 & 88,0 & 12 & 85,7 & 9 & 69,2 & 138 & 86,3 \\
\hline - Rutina no manual & 8 & 5,0 & 8 & 6,0 & 2 & 14,3 & 4 & 30,8 & 14 & 8,8 \\
\hline - Pequeña burguesía & । & 0,6 & 0 & 0,0 & 0 & 0,0 & 0 & 0,0 & 0 & 0,0 \\
\hline - Trabajador independiente & 17 & 10,6 & 5 & 3,8 & 0 & 0,0 & 0 & 0,0 & 5 & 3,1 \\
\hline - Trab. manual cali cado & 28 & 17,5 & 3 & 2,3 & 0 & 0,0 & 0 & 0,0 & 3 & 1,9 \\
\hline - Trab. manual no cali cado & 55 & 34,4 & 0 & 0,0 & 0 & 0,0 & 0 & 0,0 & 0 & 0,0 \\
\hline - Pequeño prop. agrícola & 18 & 11,3 & 0 & 0,0 & 0 & 0,0 & 0 & 0,0 & 0 & 0,0 \\
\hline - Trabajador agrícola & 15 & 9,4 & 0 & 0,0 & 0 & 0,0 & 0 & 0,0 & 0 & 0,0 \\
\hline Total & 160 & 100 & 133 & 100 & 14 & 100 & 13 & 100 & 160 & 100 \\
\hline
\end{tabular}

* Comprende solamente aquellas personas que obtuvieron título profesional universitario ( I 60 en total).

** El origen se re ere a la categoría ocupacional del padre cuando el entrevistado tenía I 4 años y el destino evidencia la categoría ocupacional actual del entrevistado.

Fuente: Elaboración propia (Cantero, 2005/2006).

Indudablemente, la educación es un factor clave en el proceso de movilidad ocupacional y social, pues las cifras evidencian que del total de personas que han accedido a la Universidad en estas comunas, independientemente de su origen social - categoría ocupacional del padre - y grupo étnico, hoy se ubican en las categorías ocupacionales de mayor connotación social: Clase de Servicio (86,3\%), Clase de Rutina No Manual (8,8\%), en tanto que tan sólo 
un $5 \%$ se desenvuelve en trabajos independientes y manuales calificados (ver última columna de la Tabela 4). Sin embargo, del total de personas con estudios universitarios, un $83,1 \%$ es de origen no mapuche ( 133 personas) y un $16,9 \%$ (27 encuestados), de origen mapuche (8,8\% mapuche y $8,1 \%$ mestizo). Estas cifras evidencian la desigualdad educacional existente por origen étnico.

\section{MOVILIDAD EDUCACIONAL Y CAMBIOS EN LA ESTRUCTURA SOCIAL POR ORIGEN ÉTNICO}

En nuestro país habitan diferentes pueblos indígenas ${ }^{8}$, siendo el mapuche el que abarca el mayor porcentaje (87,3\% del total étnico) y cuya población asciende a 604.349 personas, lo que equivale a un 4\% del total país. La Araucanía es la región que concentra la mayor población Mapuche $(23,3 \%)$ lo que equivale a 202.970 personas, destacando las comunas de Temuco, Padre Las Casas y Nueva Imperial, localidades que presentan la mayor proporción de población mapuche y no mapuche de la región (Chile, 2002a).

Estudios de Mideplan (Chile, 2002) evidencian que existen signi cativas diferencias en materia de ingresos y educación entre indígenas y no indígenas.

A nivel nacional, la tasa de analfabetismo de la población indígena supera en más del doble $(8,4 \%)$ a la tasa de analfabetismo de los no indígenas $(3,8 \%)$. En el mismo sentido, la población no indígena presenta mayores niveles educacionales que los alcanzados por la población indígena. Además, la proporción de personas indígenas que completan su enseñanza superior es menos de un tercio de la proporción de personas no indígenas en la misma situación (Chile, 2002).

Estas desigualdades educativas se podrían explicar en gran medida por las desigualdades de ingreso y los niveles de pobreza existentes en la sociedad chilena, además de otros factores que provienen del entorno sociocultural y familiar del individuo. De hecho, la principal causa a que aluden los entrevistados para no culminar sus estudios superiores es la económica (con más del 50\% de las preferencias), seguida por razones familiares (17\%) y laborales (16\%).

8. Como principales etnias indígenas en Chile, la Ley 19.253 reconoce a la Mapuche, Aymará, Rapa Nui o Pascuense, Atacameños o Lican Antay, Quechuas, Collas, Kawashkar o Alacalufe y Yamana o Yagan (Chile, 2002). 
Es indudable que la calidad de la educación recibida tanto en educación básica como media, son determinantes para acceder a la Universidad; sin embargo, aun cuando no existiesen desigualdades educacionales en la enseñanza escolar (y se tuviese por ejemplo, acceso a un proceso de enseñanza igualitaria), de todas formas se generarán diferencias tanto en rendimiento escolar como en el acceso a la Universidad. Una de las razones de estas diferencias - en que pese a su permanencia, ha disminuido su impacto por las políticas públicas de la última década - se explicaría por la relación existente entre pobreza y desnutrición, pues tal como lo plantea Debraj Ray (2002), cuando una persona tiene una baja renta, le resulta difícil adquirir un nivel suficiente de consumo de alimentos y de nutrientes para ella misma y para su familia, lo que puede ocasionar en los niños debilidad muscular, mayor vulnerabilidad a enfermedades e infecciones, pudiendo afectar también sus capacidades cognitivas. Otra razón tiene que ver con las características demográficas del hogar de origen; en general, las familias pobres o de menores recursos suelen tener una elevada proporción de personas dependientes (niños), siendo más numerosas que una familia media, lo que dificulta el proceso de nutrición y educación de sus integrantes en la infancia (Ray, 2002); en este sentido, el tamaño de la familia puede ser tanto una causa de la pobreza como un efecto. Las familias mayores, especialmente las que tienen más hijos, tienden a tener una renta per cápita más baja debido simplemente a que son más los que no trabajan, lo que estimula el trabajo infantil y el peligro, especialmente en las niñas, de quedar embarazadas tempranamente tratando de salir de la pobreza por la vía del matrimonio o simplemente por la mayor vulnerabilidad social en que se encuentran. Hay otras razones, como, por ejemplo, la descontextualización entre lo que se enseña, cómo se enseña y la lengua en que se enseña en la escuela y los contenidos culturales, pedagogía y lengua de la sociedad comunitaria local.

En el caso del estudio, para detectar si existen diferencias significativas en materia educacional por etnia, se han clasificado a los encuestados en dos grandes grupos: no mapuche y mapuche. A su vez el grupo mapuche se ha subdividido en dos subgrupos: mapuche puro y mapuche mestizo. El mestizaje (mezcla chileno-mapuche) es una categoría interesante de analizar, porque engloba dos culturas o realidades diferentes, motivo por el cual se ha separado de la categoría "mapuche" (Tab. 5). Llamaremos indistintamente a los "mestizos" también como "mezcla", debido a que consideramos una cierta predominancia 
mapuche - por el auto-referencia general de muchos mapuche al considerarse como tales, aunque tengan un sólo apellido mapuche- pero mezclado con no mapuche (chilenos o de otras etnias o culturas).

\section{TABELA 5}

NIVEL EDUCACIONAL SEGÚN ETNIA O RAZA ORIGINARIA*, COMUNAS EN ESTUDIO, IX REGIÓN (CIFRAS EN PORCENTAIES)

\begin{tabular}{lcccccc}
\hline & \multicolumn{2}{c}{ Mapuche } & \multicolumn{2}{c}{ Mapuche mestizo } & \multicolumn{2}{c}{ No mapuche } \\
\cline { 2 - 7 } & Padre & Hijo & Padre & Hijo & Padre & Hijo \\
\hline Sin educación & 22,4 & 1,5 & 9,6 & 0,0 & 3,8 & 0,0 \\
\hline Básica incompleta & 40,3 & 22,4 & 27,4 & 13,5 & 13,2 & 2,8 \\
\hline Básica completa & 25,4 & 13,4 & 28,8 & 5,4 & 26,4 & 5,6 \\
\hline Media incompleta & 1,5 & 16,4 & 9,6 & 16,2 & 7,9 & 7,8 \\
\hline Media completa & 10,4 & 19,4 & 12,3 & 35,1 & 33,1 & 25,3 \\
\hline Superior incompleta & 0,0 & 4,5 & 1,4 & 8,1 & 2,1 & 11,4 \\
\hline Superior completa & 0,0 & 22,4 & 11,0 & 21,6 & 13,5 & 47,1 \\
\hline Total (\%) & 100 & 100 & 100 & 100 & 100 & 100 \\
\hline Total (No) & 67 & 67 & 73 & 74 & 341 & 359 \\
\hline
\end{tabular}

* Sólo 48 I encuestados (hijos) declararon el nivel educacional del padre o jefe de hogar. Fuente: Elaboración propia (Cantero, 2005/2006).

Las cifras ponen de relieve que la etnia mapuche posee mayoritariamente estudios básicos y medios, siendo muy pocos los encuestados que acceden a educación superior. Una situación contraria se observa en los encuestados no mapuche, donde los estudios se concentran mayoritariamente en medios y superiores.

Al analizar el mestizaje (mezcla chileno-mapuche), los datos reflejan tendencias educacionales que toman aspectos de las dos razas, pues por una parte hay menos personas con estudios primarios en comparación a la etnia mapuche, y hay más personas con estudios medios y superiores, pero aún por debajo de lo que sucede con las personas no mapuche. Por lo tanto, los datos son concordantes con lo que muestran las diversas encuestas Casen en términos de desigualdades educacionales. 
Al analizar la movilidad educacional intergeneracional por etnia (Tabela 6), se observa que las tasas de movilidad educacional entre no mapuche, mapuche y mestizos son muy similares, destacando la etnia mapuche con la tasa de movilidad educacional más elevada $(77,6 \%)$ y la tasa de movilidad descendente más baja (3\%).

TABELA 6

MOVILIDAD EDUCACIONAL POR ORIGEN ÉTNICO, COMUNAS EN ESTUDIO, IXREGIÓN

\begin{tabular}{lrrr}
\hline & No mapuche & Mapuche & Mestizo \\
\hline - Movilidad ascendente & 72,8 & 77,6 & 72,6 \\
- Movilidad descendente & 4,8 & 3,0 & 9,6 \\
- Inmovilidad & 22,4 & 19,4 & 17,8 \\
\hline
\end{tabular}

Fuente: Elaboración propia (Cantero, 2005/2006).

La alta movilidad educacional intergeneracional ascendente que ha experimentado la etnia mapuche se debe a los bajos niveles educacionales de los padres. Tal como se muestra en la tabela 5 , un $88 \%$ de los padres de esta etnia posee un nivel educacional básico o inferior (un 22\% no posee ningún tipo de instrucción), en tanto que los hijos se han movido a la educación media y un $22 \%$ de ellos ha culminado su educación superior.

Los mayores niveles educacionales que han logrado los "hijos" respecto a sus "padres" les ha permitido acceder a clases ocupacionales de mayor connotación social, como la Clase de Servicio y Rutina No Manual. Estos cambios ocupacionales se han dado para todos los orígenes étnicos.

Más en detalle, se puede ver que las posiciones sociales que hoy ocupan los descendientes mapuche difieren mucho de las posiciones que ocupaban sus padres en las clases ocupacionales (Tab. 7). Más aún, los datos muestran que los padres de los encuestados mapuche se desenvolvían mayoritariamente en labores relacionadas con la agricultura y trabajos manuales de baja calificación, sin ninguna participación en las clases ocupacionales de más alta connotación social. Sin embargo, actualmente existe un creciente porcentaje de esta etnia que ocupa posiciones importantes en la sociedad chilena, en ocupaciones relacionadas con la Clase de Servicio (17,9\%) y de Rutina no Manual (4,5\%). 
TABELA 7

CAMBIOS INTERGENERACIONALES EN LA ESTRUCTURA SOCIAL POR ORIGEN ÉTNICO (CIFRAS EN PORCENTAJES)

\begin{tabular}{lccccccc}
\hline \multirow{2}{*}{ Categorías sociales } & \multicolumn{2}{c}{ Mapuche } & \multicolumn{2}{c}{ Mestizo } & \multicolumn{2}{c}{ No mapuche } \\
\cline { 2 - 7 } & Padre & Hijo & Padre & Hijo & Padre & Hijo \\
\hline - Clase de servicio & 0,0 & 17,9 & 8,1 & 14,9 & 7,2 & 36,2 \\
- Rutina no manual & 0,0 & 4,5 & 0,0 & 9,5 & 3,6 & 9,2 \\
- Pequeña burguesía & 0,0 & 0,0 & 0,0 & 0,0 & 0,3 & 1,4 \\
- Trabajadores independientes & 3,0 & 0,0 & 0,0 & 6,8 & 7,2 & 10,3 \\
- Trabajadores manuales cali cados & 4,5 & 13,4 & 9,5 & 16,2 & 13,1 & 12,8 \\
- Trabaj. man. no cali cados & 22,4 & 28,4 & 37,8 & 44,6 & 45,1 & 27,0 \\
- Pequeños propietarios agrícolas & 13,4 & 8,9 & 14,9 & 5,4 & 8,1 & 1,1 \\
- Trabajadores agrícolas & 56,7 & 26,9 & 29,7 & 2,7 & 15,3 & 1,9 \\
\hline & Total (\%) & 100 & 100 & 100 & 100 & 100 & 100 \\
\hline & 67 & 67 & 74 & 74 & 359 & 359 \\
\hline
\end{tabular}

Fuente: Elaboración propia (Cantero, 2005/2006).

Evidentemente, las personas de origen mapuche que hoy ocupan cargos de mayor trascendencia social son aquellas con mayor preparación educacional, con estudios técnicos, profesionales y universitarios. Por lo tanto, se puede decir que la sociedad chilena ha ido abriendo espacios para una mayor cantidad y variedad de sus miembros, reduciendo las discriminaciones sociales y facilitando que el "logro" prime más que la "adscripción".

Además, los cambios entre clases ocupacionales "padres-hijos" no sólo han existido en la etnia mapuche, sino también en los grupos no mapuche y mestizos, tal como se muestra en la tabela 7. En ambos grupos se observa que los padres se desenvolvían mayoritariamente en trabajos agrícolas y manuales de baja calificación, en tanto que los hijos, gracias a sus mayores logros educacionales, han podido ascender en la escala social, ganando cada vez más espacio en las Clases de Servicio y de Rutina No Manual. Sin embargo, esta situación se ha dado con mayor fuerza en el grupo no mapuche y con menos fuerza en el grupo mestizo, dado que en este último se visualiza una migración de trabajos agrícolas a trabajos manuales urbanos de baja calificación. Este hecho se explicaría, más que por una situación discriminatoria racial, por los mayores 
logros educacionales que se observan en el grupo no mapuche por sobre el "grupo mestizo". Pese a lo anterior, es claro que la sociedad chilena ha venido evolucionando en términos de estructura ocupacional.

Por otra parte, pese a los importantes cambios educacionales y ocupacionales que ha experimentado el pueblo mapuche, al compararlo con los otros grupos étnicos del estudio, se pone de manifiesto que todavía persiste un gran apego a actividades relacionadas con la agricultura (gráfico I). El grupo mestizo se ha desplazado a actividades ocupacionales más urbanas aunque de baja calificación (trabajos manuales no calificados), mientras que el grupo no mapuche acentúa su participación en la clase de servicio, gracias a sus mayores logros educacionales.

\section{GRÁFICO I \\ PARTICIPACIÓN ÉTNICA POR CLASES SOCIALES}

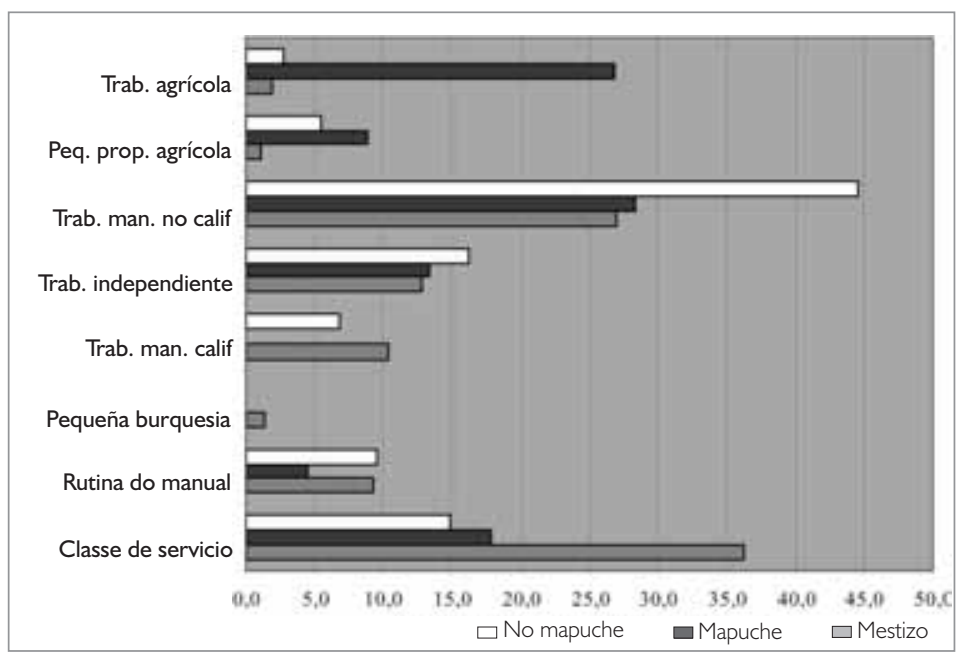

Fuente: Elaboración propia (Cantero, 2005/2006).

La alta permanencia de la etnia mapuche en las ocupaciones agrícolas se explica porque un significativo porcentaje (más de un 43\%) de los entrevistados habita en la zona rural, donde se registran los más bajos niveles educacionales. Los datos del estudio evidencian que en la zona rural la educación se centra en los niveles básicos y medios, siendo muy pocos los individuos que acceden a 
la educación superior. De hecho, tan sólo un 1,5\% de las personas de origen mapuche que han accedido a educación superior se ubica en esta zona.

Varias razones explican los bajos logros educacionales que se registran en la zona rural; hay razones curriculares, pedagógicas, culturales, docentes, sin embargo nos concentraremos en algunas causales sociales. En primer término, se debe mencionar que en ella se concentran los mayores índices de pobreza e indigencia, lo que impide que los jóvenes continúen estudios superiores en las zonas urbanas, viéndose en la necesidad de abandonar tempranamente sus estudios para apoyar el sustento familiar, ya sea empleándose en un trabajo urbano de baja calificación o para dedicarse al cultivo de la tierra. Esta carencia de capital humano (preparación educacional), a largo plazo, impide moverse hacia clases urbanas de mayor calificación, debiendo permanecer en su clase de origen (lo que sucede con la etnia mapuche). En segundo término, se debe recordar que en la zona rural existe una alta prevalencia de la herencia, donde los recursos son fácilmente transferibles entre generaciones, como es el caso de la tierra o la propiedad. Por último, cabe mencionar que en la zona rural no existe la posibilidad de retomar fácilmente los estudios como sucede en el área urbana, donde los individuos tienen más oportunidades de continuar sus estudios aún en edad adulta. En este sentido, las cifras evidencian que del total de personas entrevistadas en la zona urbana (4I7), un 21, I\% de ellas se encuentra actualmente realizando algún tipo de estudio (carreras técnicas, diplomados, postítulos, magíster y doctorados, entre otros); en tanto que en la zona rural, esta cifra sólo alcanza a un 1,2\%.

Esta última situación ha generado una emigración de personas desde las zonas rurales hacia las urbanas, reduciendo el porcentaje poblacional que habita en las zonas rurales y aumentando la densidad poblacional en las urbanas. A nivel País, según datos del Censo 2002, en 1992 un 83,5\% de la población residía en áreas urbanas y un 16,5\% en zonas rurales; en el año 2002, el porcentaje de personas que habita en áreas urbanas es de $86,6 \%$, en tanto que en las zonas rurales vive el 13,4\% de la población total, lo cual evidencia un aumento sustantivo del porcentaje urbano en desmedro del rural. Al respecto, los datos del estudio muestran que del total de encuestados en la zona urbana, un 22, I \% habitaba en la zona rural a los 14 años. Indudablemente, las mayores oportunidades educacionales con que cuentan las personas en la zona urbana, el acceso a mejores ocupaciones y mayores ingresos, la modernización rural 
generados por el modelo de desarrollo imperante, han repercutido en estos cambios.

\section{CONCLUSIONES}

El análisis de la estratificación social se puede realizar desde diversas perspectivas, pero - como ya señalamos - una de las formas más utilizadas es la ocupación, dado que permite una determinada visión de la estructura social. La ocupación es un "proxy" relativamente adecuado del acceso de los individuos a la educación y al ingreso, que son dos dimensiones que intervienen en el sistema de estratificación social.

La educación juega un papel central como mecanismo promotor de oportunidades ocupacionales, dado que mientras mayor sea el nivel educacional de un individuo más posibilidades tiene de ascender socialmente, independientemente de su origen social.

En especial, la educación superior es un factor clave de ascenso social, pues permite que todas las personas que accedan a ella tengan la oportunidad de conseguir mejores ocupaciones y percibir más altos ingresos, mejorando así su calidad de vida. El estudio evidencia que más del $85 \%$ de las personas con estudios universitarios completos hoy se desenvuelven en actividades vinculadas a la clase de servicio, independientemente de su origen social y étnico.

En el tema de la movilidad educacional, el estudio muestra una significativa tasa de movilidad educacional ascendente, pues más del 70\% de los encuestados superaron los niveles educacionales de sus padres, poco más de $20 \%$ lo mantiene, y tan sólo un 5\% desciende educacionalmente. Por lo tanto, en estas comunas ha existido un cambio intergeneracional de notable importancia en el tema educacional, ya que mientras la educación de los padres se concentra en los niveles básicos y medios, los niveles educacionales de los hijos se acentúan significativamente en el nivel medio e incluso superior.

Este cambio educacional, sin duda, está relacionado con el cambio intergeneracional que se ha dado en la estructura social, lo cual se refleja en el índice de disimilitud que alcanza en las comunas en estudio a un 35,8\%. Los datos muestran una ampliación de la clase de servicio y de rutina no manual y una reducción de la clase de trabajadores manuales no calificados, pequeños propietarios agrícolas y trabajadores agrícolas. Son justamente los individuos 
provenientes de estas últimas clases los que se han movido ascendentemente a la clase de servicio, gracias a los mayores logros educacionales y en especial a la incidencia de la educación superior. La migración que se ha dado desde las clases agrícolas y de escasa calificación hacia las clases urbanas y de mayor calificación, estaría evidenciando una pérdida de importancia relativa de la "campesinización".

En el tema étnico, si bien se han reducido las desigualdades educacionales históricas, éstas siguen existiendo, afectando con mayor rigor a los descendientes mapuche y mestizos. A pesar de los mayores logros educacionales de la etnia mapuche y mestizos y del acceso a ocupaciones de mayor connotación social, aún queda mucho por hacer en cuanto a pobreza, indigencia, inequidades y oportunidades educativas, principalmente en la zona rural, pues es aquí donde se concentra la mayor proporción de población mapuche pobre con identidad.

De lo expuesto se podría pensar que las políticas de educación para la población rural y de educación intercultural bilingüe democráticas, en el contexto de otros programas educacionales (infraestructura, alimentación y salud escolar, profesionalización docente, entre otros) han logrado contribuir a una movilidad social al interior de la educación básica y desde ésta a la media; sin embargo, por haberse concentrado en el nivel básico, no han conseguido influir sustancialmente en el paso del nivel medio al superior. Es decir, para los hijos e hijas del pueblo mapuche, las acciones públicas han promovido la movilidad básica-rural a la media-urbana y en menor medida de la media-urbana a la superior.

El estudio demuestra en lo regional y en lo inter-étnico la desigualdad que persiste en la aplicación de derechos en la educación entre mapuche y no mapuche y que ha sido estudiada por diversas investigaciones referidas a derechos humanos, índices de desarrollo humano e infancia, derecho a la educación, desigualdades estructurales del sistema de educación superior que no han conseguido ser superadas por los avances de las políticas educacionales de los últimos años (Williamson, 2006).

La investigación evaluativa de la EIB muestra que hay avances en el acceso de alumnos y alumnas indígenas al nivel de pregrado apoyado por la Beca Indígena, en cierta medida hay mayor permanencia durante los estudios, mayor egreso y titulación y continuación de estudios de postgra- 
do gracias a programas y políticas de acción afirmativa universitaria, pero que ello no es suficiente frente a la situación de exclusión de la mayoría de los jóvenes mapuche de La Araucanía y del país (Williamson, 2006). En varios casos que observamos o estudiamos, de Liceos de enseñanza media humanista-científica y técnico-profesional, se muestra el alto nivel de desempleo (sobre todo femenino) o de ingreso a empleos precarios de los egresados, con una mínima continuidad de estudios superiores a nivel técnico o universitario.

Sin duda que sin cambios estructurales en las relaciones inter-étnicas, entre estado, sociedad civil y pueblos indígenas y en las relaciones económicas y ambientales en relación al respeto por la Ley Indígena; sin cambios institucionales como la aprobación del Convenio 169 de la Organización Internacional del Trabajo - OIT -, la derogación o modificación sustancial de la Ley Orgánica Constitucional de Enseñanza y de Municipalidades y de la Constitución del país, será muy difícil revertir esta tendencia de aumento de la movilidad social, pero en los marcos estructurales de la desigualdad étnica, social y de ingresos. Sin embargo, aun en este contexto, la educación continúa jugando un papel central en las posibilidades de que los jóvenes mapuche y mestizos puedan mejorar a lo menos sus condiciones de ingreso por la vía de mejorar sus posibilidades de movilidad social al aumentar sus estudios.

Mejorar la calidad de la educación bajo principios de igualdad (derechos humanos), diversidad (pluralismo) y solidaridad (interculturalidad); contextualizarla curricular y pedagógicamente; ampliar la participación social y familiar bajo principios de gestión participativa en educación; resolver los problemas de financiación del sector municipal; asumir una concepción territorial de la educación donde comunidades, comunas y regiones aprenden interculturalmente; impulsar políticas y programas de acción afirmativa; éstas son algunas de las medidas que deben adoptarse para avanzar hacia una mayor igualdad entre mapuche, mestizos y no mapuche en sus oportunidades de mejorar sus ingresos, ocupaciones y condiciones de vida con identidad, en la perspectiva de promover un desarrollo territorial más inclusivo, democrático, multicultural, con crecimiento sostenido y distribución justa de la riqueza generada por todos los trabajadores y productores del pasado y presente del territorio. 


\section{REFERÊNCIAS BIBLIOGRÁFICAS}

ALAMOS, P. Educación: con todos es mejor. In: DÍAZ-ROMERO, P. Caminos para la inclusión en la educación superior: Chile. Santiago: Fundación Equitas, 2006. p.24-32.

ATRIA, R. Estructura ocupacional, estructura social y clases sociales. Santiago: División de Desarrollo Social, Cepal, 2004. (Políticas Sociales, n.96)

CANTERO, V. Educación y movilidad social: un estudio empirico aplicado a las famílias mapuche y no mapuche de las comunas de: Temuco, Padre las Casas y Nueva Imperial. Temuco: Universidad de la Frontera, 2005/2006. (Proyecto)

CARABAÑA, M. J. Educación y movilidad social. In: SEMINARIO EL ESTADO DE BIENESTAR EN ESPAÑA. Documento de ponencia. Barcelona: U. de Menendez Pelayo, p. 18 - 19 , Dic. 2003.

CHILE. Instituto Nacional de Estadística. Ministerio de Plani cación. Análisis de la VIII encuesta de caracterización socioeconómica nacional (Casen 2000). Documento n. I 4. Etnias y Pobreza en Chile. Santiago, 2002.

. Censo 2002: síntesis de resultados, características demográ cas población total. Santiago, 2002a.

. Informe Regional Región de la Araucanía. Encuesta de Caracterización Socioeconómica Nacional, Casen 2003. División Social, Gobierno de Chile, 2003.

. Pobreza y distribución del ingreso en las regiones: Región de la Araucanía. Encuesta de caracterización socioeconómica nacional, Casen 2003. Santiago: División Social, Gobierno de Chile, 2004.

CORBO, V. Educación, equidad y crecimiento. Economía y Negocios, Dic.2002. Disponível em: www.economiaynegocios.cl. Acesso em: jul. 2007.

ERIKSON, R.; GOLDTHORPE, J. H. The Constant flux: a study of class mobility in industrial societies. Oxford: Oxford University, 1993.

FILGUEIRA, C. La Actualidad de viejas temáticas: sobre los estudios de clase, estrati cación y Movilidad social en América Latina. Santiago: División de Desarrollo Social, Cepal, 200 I. (Políticas Sociales, n.5I)

GERMANI, G. Estrategia para estimular la movilidad social. Desarrollo Económico, v. I, n.3, I96I.

HOPENHAYN, M. Educación, comunicación y cultura en la sociedad de la información: una perspectiva latinoamericana. Revista de la Cepal, n.8I, p.175-193, Dic. 2003. Revisión de políticas nacionales de educación: Chile. Santiago, 2004. 
PÉREZ BRIGNOLI, H. Educación, capital humano y movilidad social en Costa Rica: un primer análisis de los datos del Censo de 2000. San José: Centro Centroamericano de Población de la Universidad de Costa Rica, 2004. p.27I -285. (Proyecto Estado de la Nación e Instituto de Estadística y Censo)

RAY, D. Economía del desarrollo. Madrid: Antoni Bosch, 2002.

TORCHE, F; WORMALD, G. Estratificación y movilidad social en Chile: entre la adscripción y el logro. Santiago, Cepal, Dic. 2004. (Políticas Sociales, n.98)

UNESCO. Comisión Económica para américa Latina y el Caribe. Educación y conocimiento: eje de la transformación productiva con eqüidad. Santiago: Naciones Unidas, 1992.

UNICEF; CHILE, Ministerio de Plani cación. Índice de infancia, Chile 2002: una mirada comunal y regional. Santiago, 2003.

WINKLER, D. R.; CUETO, S. Etnicidad, raza, género y educación en América Latina. Santiago: Preal, 2004.

WILLIAMSON, G. Gobierno universitario: democratización para una acción a rmativa más e caz. In: DIAZ-ROMERO, P. (Ed.). Caminos para la inclusión en la educación superior: Chile. Santiago: Fundación Equitas, 2006. p.293-320.

Recebido em: agosto 2007

Aprovado para publicação em: novembro 2008 